\title{
Nonperiodic Orbit Sums in Weyl's Expansion for Billiards
}

\author{
Wei-Mou Zheng \\ Institute of Theoretical Physics, Academia Sinica, Beijing 100080, China
}

\begin{abstract}
Weyl's expansion for the asymptotic mode density of billiards consists of the area, length, curvature and corner terms. The area term has been associated with the so-called zero-length orbits. Here closed nonperiodic paths corresponding to the length and corner terms are constructed.
\end{abstract}

PACS numbers: 05.45., 03.65.Sq

\section{Introduction}

The asymptotic mode density appears in various branches in physics. In a very early analysis of the density of eigenmodes for a cavity with reflecting walls, Weyl proved that the leading term is proportional solely to the volume of the domain, and independent of the shape [1]. Since then terms which account for the surface, shape and connectivity of the domain were found to refine asymptotic expansions [2, 3, 4. inside a simply connected domain of the Euclidean plane, the two-dimensional version of Weyl's expansion reads

$$
\rho(E) \sim \frac{\mathcal{A}}{4 \pi}-\frac{\mathcal{L}}{8 \pi \sqrt{E}}+\left[\frac{1}{12 \pi} \oint c(s) d s+\frac{1}{24} \sum_{i}\left(\frac{\pi}{\alpha_{i}}-\frac{\alpha_{i}}{\pi}\right)\right] \delta(E),
$$

where Dirichlet boundary conditions are used, units are set at $2 m=\hbar=1$, the boundary is characterized by the arc length $s$, and $\mathcal{A}, \mathcal{L}, c(s), \alpha_{i}$ are the area, the total length, curvature and inner angle of corner, respectively.

Most studies of eigenvalue densities are based on the Green's function technique. Mathematical asymptotic theory involves the Tauberian theorems from the theory of Laplace transforms. Balian and Bloch worked with energy Green's functions. Without requiring Tauberian theorems, they obtained a multiple reflection expansion for Green functions. The use of curvilinear coordinates was also included in their discussions.

The area term has the simple meaning that the probability for a system to be in a particular subset of phase space is proportional to the volume of the subset. This term has been associated with the so-called zero-length orbits in the semiclassical theory [5].

The periodic orbit theory of Gutzwiller, known as the trace formulas, relates the fine details of oscillating density of states with classical periodic orbits [6, 7]. A trace formula has been derived for integrable systems by Berry and Tabor [8]. Other extensions of the Gutzwiller theory have been developed [9, 10]. However, not much attention has been drawn to the role of nonperiodic orbits. Here we shall relate some nonperiodic orbits to Weyl's expansions. In Sec. II a family of closed nonperiodic orbits of single reflection is given to account for the length term. In Sec. III orbits contributing to the corner term are examined. Finally, some remarks are made in the last section.

\section{The length term}

Using the stationary method, Gutzwiller derived from Feynman's path integral the approximate propagator from $\mathbf{r}$ to $\mathbf{r}^{\prime}$ in the time difference $t[6]$

$$
K_{s c}\left(\mathbf{r}, \mathbf{r}^{\prime} ; t\right)=\sum_{\text {cl.tr. }}(2 \pi i)^{-N / 2} \sqrt{|\operatorname{det} C|} \exp \left[i R\left(\mathbf{r}, \mathbf{r}^{\prime} ; t\right)-i M \pi / 2\right],
$$


where $R$ is Hamilton's principal function, $M$ the phase index obtained by counting the number of conjugate points along each classical path over which the summation is taken, $N$ the dimensionality, and $C$ the matrix of the negative second variations of $R$

$$
C_{i j}\left(\mathbf{r}, \mathbf{r}^{\prime} ; t\right)=-\frac{\partial^{2} R}{\partial r_{i} \partial r_{j}^{\prime}}
$$

By converting time to energy, the propagator is transformed to Green's function as

$$
G\left(\mathbf{r}, \mathbf{r}^{\prime} ; E\right)=i^{-1} \int_{0}^{\infty} d t K\left(\mathbf{r}, \mathbf{r}^{\prime} ; t\right) \exp (i E t) .
$$

By using the propagator (2), after integrating over time again by the method of stationary phase, Gutzwiller's semiclassical approximation to Green's function is given by

$$
G_{s c}\left(\mathbf{r}, \mathbf{r}^{\prime} ; E\right)=\frac{2 \pi}{(2 \pi i)^{(N+1) / 2}} \sum_{\text {cl.tr. }} \sqrt{|D|} \exp \left[i S\left(\mathbf{r}, \mathbf{r}^{\prime} ; E\right)-i \mu \pi / 2\right],
$$

where $S$ is the action integral, $\mu$ is again the phase index, i.e. the number of conjugate points, but obtained by varying the trajectory at constant energy $E$ instead of time $t$, and

$$
D=(-1)^{N} \frac{\partial^{2} S}{\partial E^{2}} \operatorname{det} C=(-1)^{N} \frac{1}{\dot{q} \dot{q}^{\prime}} \operatorname{det}\left(\frac{\partial \mathbf{p}_{\perp}^{\prime}}{\partial \mathbf{r}_{\perp}}\right),
$$

where in the last equation $\mathbf{r}_{\perp}$ is in the subspace transverse to the trajectory in the local coordinate system, and $\mathbf{p}_{\perp}$ is its conjugate momentum. Once Greeen's function is known, the density of states can be calculated by

$$
\rho(E)=-\frac{1}{\pi} \operatorname{Im} \int d \mathbf{r} G(\mathbf{r}, \mathbf{r} ; E)
$$

When the semiclassical approximation is made, the density of states is expressed in terms of classical closed orbits. The zero-length orbits contribute to the average density of states. When the method of stationary phase is again used for the integration over $\mathbf{r}$, only the periodic orbits still remain in the summation.

For a billiard, there is a family of closed orbits involving single reflection. Consider the simplest case when the boundary consists of the $x$-axis, and the inside region is the upper plane. A closed orbit of the family is an orbit going from $(x, y)$ (with $y$ positive) to $(x, 0)$ and then returning back to $(x, y)$. The calculation of the Jacobian $\partial r_{\perp} / \partial p_{\perp}^{\prime}$ in the expression (6) for D is given in the appendix. From Eqs. (6), and (42), noticing that

$$
E \equiv k^{2}, \quad L \equiv 2 y, \quad \dot{q}=2 k=L / t, \quad \partial^{2} S / \partial E^{2}=-L /\left(4 k^{3}\right),
$$

we find

$$
D=1 /(8 k y), \quad|\operatorname{det} C|=1 /\left(4 t^{2}\right) .
$$

It is easy to verify that Hamilton's principal function $R$ and the action integral $S$ are

$$
R=y^{2} / t, \quad S=2 k y .
$$

From the approximation (2) for the propagator, we have

$$
K_{\text {co }}=-\frac{1}{4 i \pi t} \exp \left(i \frac{y^{2}}{t}\right),
$$

where subscript 'co' stands for 'closed orbits'. By using the formula for Hankel's function

$$
H_{\nu}^{(1)}(x z)=\frac{i^{-\nu-1}}{\pi} z^{\nu} \int_{0}^{\infty} \exp \left[\frac{1}{2} i x\left(t+\frac{z^{2}}{t}\right)\right] t^{-\nu-1} d t,
$$


from Eq. (4) Green's function is obtained as

$$
G_{\mathrm{co}}(\mathbf{r}, \mathbf{r} ; E)=-\frac{1}{4 i} H_{0}^{(1)}(2 k y) .
$$

By means of the relation (7), its contribution to the density of states may be estimated by using

$$
\int_{0}^{\infty} d z z^{\mu} H_{\nu}^{(1)}(a z)=\frac{1}{\pi} 2^{\mu} i^{\mu-\nu} a^{-\mu-1} \Gamma\left(\frac{1+\mu+\nu}{2}\right) \Gamma\left(\frac{1+\mu-\nu}{2}\right)
$$

to be

$$
-\int d x \int_{0}^{\infty} d y \frac{1}{4 i} H_{0}^{(1)}(2 k y)=-\frac{\mathcal{L}}{8 \pi \sqrt{E}},
$$

which gives exactly the length term in Weyl's expansion (11).

If we estimate Green's function directly from Eq. (5), the result becomes

$$
G_{\mathrm{co}}^{\prime}=-\frac{1}{4 i \sqrt{\pi k y}} \exp (2 i k y)
$$

which is consistent with the above $G_{\mathrm{co}}$ in the asymptotic approximation. However, its contribution to the density of states is $\mathcal{L} /(4 \sqrt{2 E} \pi)$, which is different from the expected result by a factor of $\sqrt{2}$. This situation is similar to that of zero-length orbits. Since integration over small $y$ contributes significantly, we have to use the uniform approximation with Hankel's function.

When a bounce takes place at a position $s$ of the boundary with curvature $c(s)$, the closed orbit along the normal to the boundary still exists. Set the coordinate system with the origin at the bounce point and

the $y$-axis along the normal directing towards the inside of the billiard. By using Eqs. (39) and (40), the counterparts of quantities (8) are found to be

$$
D=1 /[8 k y(1-c y)], \quad|\operatorname{det} C|=1 /\left[4 t^{2}(1-c y)\right],
$$

which gives the same length term.

\section{The corner term}

In the previous section we have considered closed orbits of single reflection. For a corner with an acute angle there is a family of closed orbits with double reflection. We shall first examine an acute corner, and then extend the analysis to an obtuse corner.

\subsection{Acute corners}

For a corner with an acute angle $\alpha$ we may construct a closed orbit of double reflection as follows. Let us denote by $O$ the vertex of the corner, and by $O A$ and $O B$ its two sides. Suppose that the mirror image of $O A$ with respect to $O B$ is $O A^{\prime}$, and the image of $O B$ with respect to $O A^{\prime}$ is $O B^{\prime}$. In this way we get the first and second images of the original corner, and a given point $Q$ is then mapped to $Q_{2}$ in the corner $A^{\prime} O B^{\prime}$, as shown in Fig. 1. The straight line $Q Q_{2}$, after mapping its segments back into the original corner, gives the closed orbit looked for.

Denote by $r=|O Q|$ the length of $O Q$. The length of the closed orbit is then

$$
\left|\mathrm{QQ}_{2}\right|=2 r \sin \alpha \text {. }
$$

From Eqs. (42) and (2), we have

$$
K_{\mathrm{co} 2}=\frac{1}{4 i \pi t} \exp \left(i \frac{(r \sin \alpha)^{2}}{t}\right)
$$


which, from Eqs. (伍) and (7), leads to

$$
\rho_{\mathrm{co}}=\frac{\alpha}{8 \pi \sin ^{2} \alpha} \delta(E)
$$

where we have used formula $(13)$ and have written $E$ as $E+i \epsilon$ to give

$$
\frac{1}{E+i \epsilon}=\mathcal{P} \frac{1}{E}+\frac{\pi}{i} \delta(E)
$$

The corner term in Weyl's expansion for the rectangular corner is estimated to be $1 / 16$, in agreement with the value given by expression (19).

Expression (19) is not the same as the corner term in Weyl's expansion (11). A corner gives a correction to the length term due to the restriction on the domain of integration. Set the vertex $\mathrm{O}$ at the origin, and side OA along the $x$-axis. The change in the domain of integration

$$
\int d x \int_{0}^{\infty} d y \rightarrow \int d x \int_{0}^{\gamma x} d y, \quad \text { with } \quad \gamma=\tan \alpha
$$

gives the correction to the density of states

$$
\delta \rho=-\frac{2}{\pi} \operatorname{Im} \int_{0}^{\infty} d y \int_{0}^{y / \gamma} d x \frac{1}{4 i} H_{0}^{(1)}(2 k y)=\frac{1}{4 \pi \gamma} \delta(E),
$$

where the factor 2 in front accounts for the two sides, and use of Green's function (12) for single reflection orbits has been made. Thus, by combining the above two corrections, the total is

$$
\rho_{\mathrm{co} 2}=\frac{1}{8 \pi}\left(\frac{\alpha}{\sin ^{2} \alpha}+2 \cot \alpha\right) \delta(E) .
$$

This is not of the same form as that in Weyl's expansion. For $\alpha=(1 / 2-\delta) \pi$ a little smaller than the rectangle, from Eq. (21) the lowest order is $1 / 16+\delta / 8$, while that from Weyl's expansion is $1 / 16+5 \delta / 24$. For very small $\alpha$, compared with $\pi /(24 \alpha)$ from expression (1), the semiclassical value is $3 /(8 \pi \alpha)$. The ratio is $9 / \pi^{2} \approx 0.912$.

In the above discussion we have considered only orbits of double reflection which hit the side $O B$ first. An extra factor 2 should appear for those orbits to count the two different ways of selecting orbits according to which side is hit first.

\subsection{Obtuse corners}

For an obtuse corner the above closed orbits of double reflection do not exist. A natural way of continuation has to be found. For this purpose we use the folding property of the propagator

$$
K\left(\mathbf{r}, \mathbf{r}^{\prime} ; t-t^{\prime}\right)=\int d \mathbf{r}^{\prime \prime} K\left(\mathbf{r}, \mathbf{r}^{\prime \prime} ; t-t^{\prime \prime}\right) K\left(\mathbf{r}^{\prime \prime}, \mathbf{r}^{\prime} ; t^{\prime \prime}-t^{\prime}\right),
$$

to include two-piece closed orbits. We first examine the case of an acute corner. Let us consider a twosegment broken line from $Q$ to $Q_{2}$ via some mediate point $Q^{\prime}$ shown in Fig. 1, and set $t-t^{\prime \prime}=t^{\prime \prime}-t^{\prime}=\tau$. Suppose that the polar coordinates of these three points are $\left(r, \theta_{1}\right),\left(r_{2}, \theta_{2}\right)=\left(r, 2 \alpha+\theta_{1}\right)$ and $\left(r_{0}, \theta_{0}\right)$, respectively. In similarity to Eq. (10), we may derive the propagator for each segment. By means of the folding property, we find for the broken path

$$
K\left(Q, Q_{2} \mid Q^{\prime}\right)=\frac{1}{4 i \pi \tau)^{2}} \int d \theta_{0} \exp \left\{\frac{i}{4 \tau}\left[2 r^{2}+2 r_{0}^{2}-2 r r_{0} \cos \left(\theta_{0}-\theta_{1}\right)-2 r r_{0} \cos \left(\theta_{0}-\theta_{2}\right)\right]\right\},
$$

where the integration domain for $\theta_{0}$ is determined by the constraints $0 \leq \theta_{0} \leq 3 \alpha, 0 \leq \theta_{1} \leq \alpha,\left|\theta_{0}-\theta_{1}\right| \leq \pi$ and $\left|\theta_{0}-\theta_{2}\right| \leq \pi$. At the limit of the rectangular corner, propagator (23), after integrating over $\theta_{1}$, reduces to

$$
\int_{0}^{\alpha} d \theta_{1} K\left(Q, Q_{2} \mid Q^{\prime}\right)=\frac{\pi}{2} K\left(Q, Q_{2} \mid Q^{\prime}\right)=\frac{\pi}{2}\left\{\frac{1}{2} \frac{1}{4 i \pi t} \exp \left(i \frac{r^{2}}{t}\right)\right\} .
$$


Compared with the expected form (18), $K\left(Q, Q_{2} \mid Q^{\prime}\right)$ equals the half of $K_{\mathrm{co} 2}$. It can be verified that if we use the method of stationary phase approximation in the Cartesian coordinate system for the integration involving $Q^{\prime}$ the propagator (23) would revert to (18) exactly. However, if we count the two ways of selecting orbits, the result here turns out to be better.

Generally, we may approximate the propagator between a reference point and some mediate one, say $Q$ and $Q^{\prime}$ in Fig. 1, by the semiclassical propagator involving classical paths with 0 to 2 bounces. Besides images $Q_{1}$ and $Q_{2}$, point $Q$ has two more images $Q_{-1}$ and $Q_{-2}$ obtained clockwisely, as shown in Fig. 1. The paths which contribute to the semiclassical propagator from $Q^{\prime}$ to $Q$ are straight segments $Q^{\prime} Q, Q^{\prime} Q_{1}$, $Q^{\prime} Q_{2}, Q^{\prime} Q_{-1}$ and $Q^{\prime} Q_{-2}$. The last two correspond to paths hitting side $O A$ first. Similarly, by considering images of $Q^{\prime}$, paths contributing to the propagator from $Q$ to $Q^{\prime}$ can be found. By means of the folding property, combination of these two propagators gives an approximate propagator from $Q$ to $Q$ itself, which includes $K\left(Q, Q_{2} \mid Q^{\prime}\right)$ as a part.

Calculation in the Cartesian coordinate system for the rectangular corner is rather easy. In this case both $Q_{2}$ and $Q_{-2}$ are in the third quadrant, while $Q_{1}$ and $Q_{-1}$ are, respectively, in the second and fourth quadrants. Each path arises as a length square in the exponent of the expression for propagator. Denote by $(x, y)$ and $\left(x_{0}, y_{0}\right)$ the coordinates of $Q$ and $Q^{\prime}$, respectively. There is a correspondence between closed paths and length square sums $\left[\left(x \pm x_{0}\right)^{2}+\left(y \pm y_{0}\right)^{2}\right]+\left[\left(x \pm x_{0}\right)^{2}+\left(y \pm y_{0}\right)^{2}\right]$. Here the first square bracket corresponds to the path from $Q$ to $Q^{\prime}$, and the second to the return path. A plus sign between $x$ and $x_{0}$ indicates a bounce on side $O B$, while that between $y$ and $y_{0}$ indicates a bounce on side $O A$. Thus, each path has its 'four signs' signature, which is the four signs appearing in the square sum. For example, the closed path without any bounces may be marked as ---- , which contributes to the area term. It can be verified that each of the paths -+-+ and +-+- results in

$$
\frac{1}{2} \frac{\mathcal{L}}{8 \pi \sqrt{E}}-\frac{1}{16 \pi^{2}} \delta(E)
$$

for the level density, while each of the paths,,+----+----+- and ---+ gives

$$
-\frac{1}{2} \frac{\mathcal{L}}{8 \pi \sqrt{E}}+\frac{1}{32 \pi} \delta(E)
$$

Closed paths with a total of 2 bounces, besides paths -+-+ and +-+- , are,,+--+-++-++-and --++ , each of which contributes $\delta(E) / 64$. Paths with 3 bounces are,,+++-++-++-++ and -+++ , each of which contributes $-\delta(E) / 32 \pi$. The only path with 4 bounces is ++++ , whose contribution is $\delta(E) /\left(16 \pi^{2}\right)$. The total contribution of these 16 terms recover exactly the area and length terms, and gives the corner term as

$$
\left(\frac{1}{16}-\frac{1}{16 \pi^{2}}\right) \delta(E)
$$

We see that the paths with a single bounce on both sides gives the main contribution to the corner term.

Thus, for an obtuse corner, although closed classical orbits of double reflection generally do not exist, we may still calculate the corner term from the two-piece paths of just 2 single reflections on both sides, which are made either by only one piece, or by each of the two pieces. Due to the cancellation among terms for the folding propagator the approximation keeps the main contribution. However, now the integrals involved cannot be estimated analytically, and numerical methods have to be used.

\section{Discussions}

In the above we have examined the role played by closed classical orbits with single and double reflection on the boundary for billiards. Here we make some concluding remarks.

1. So far, we have considered only the length term and the corner term, leaving the curvature term untouched. The same sign for both Dirichlet and Neumann conditions gives us a hint that orbits of double reflection is dominant. In principle, we can consider the contribution from two-piece closed 
pathes, as we did for obtuse corners. Unfortunately, even the simple case of a circle does not admit simple expressions. One way to get round the intricate situation of a circle is to consider its inscribed polygons 画.

2. A way to derive the propagator for a corner is to use curvilinear coordinates. A corner can be 'flattened' by introducing the tranformation from $(x, y) \rightarrow(u, v)$ defined by

$$
x=r \cos (\bar{\gamma} \varphi), \quad y=r \sin (\bar{\gamma} \varphi), \quad \text { with } \quad r^{2}=u^{2}+\gamma^{2} v^{2}, \quad \tan \varphi=\frac{\gamma v}{u}, \quad \gamma=\frac{\pi}{\alpha}, \quad \bar{\gamma}=\frac{\alpha}{\pi},
$$

where $\alpha$ is the inner angle of the corner as before. The Jacobian of this transformation is equal to one, which is made to keep the area and length terms unchanged. The transformed Laplacian can be derived as

$$
\Delta=\partial_{u}^{2} \partial_{v}^{2}\left(\gamma^{2}-1\right)\left(\partial_{u}^{2}-\frac{u^{2}}{r^{2}} \partial_{u}^{2}-\frac{v^{2}}{r^{2}} \partial_{v}^{2}-\frac{2 u v}{r^{2}} \partial_{u} \partial_{v}-\frac{u}{r^{2}} \partial_{u}-\frac{v}{r^{2}} \partial_{v}\right) .
$$

We now regard the product with the factor $\left(\gamma^{2}-1\right)$ as the perturbation to $\partial_{u}^{2} \partial_{v}^{2} \equiv \Delta_{0}$. The factor $\left(\gamma^{2}-1\right)$ is indeed tempting if one notes that the corner term is

$$
\frac{\pi^{2}-\alpha^{2}}{24 \pi \alpha}=\frac{1}{24}(\gamma-\bar{\gamma})=\frac{\gamma^{2}-1}{24 \gamma}
$$

By means of the perturbation expansion for the propagator the corner term can be obtained.

3. Only Dirichlet boundary conditions have been considered in the above. The extension to Neumann conditions is rather straightforward. Since there seems to be no general formula for the corner term at Neumann conditions in the literature [7], a semiclassical estimation can now be made using our approach.

4. The closed paths considered above have a zero limiting length. There are other closed orbits of a non-zero limiting length. For example, there is a continuous family of closed orbits from the diameter orbit to the equilateral triangle orbit in a circular disk. Any member of the family is an isosceles triangle with one vertex inside the circle. The role played by such orbits is worth examining.

5. We may extend our analysis to include connectivity and higher dimensionality.

Some problems are under study.

\section{Appendix: Jacobian $\partial r_{\perp} / \partial p_{\perp}^{\prime}$}

For a billiard inside a simply connected domain of the Euclidean plane, we may derive the Poincaré map from bounce to bounce in Birkhoff coordinates $(s, v)$, where $v$ is the component of the velocity in the tangent direction to the boundary right after reflection, and $s$ the arc length along the boundary. Since the absolute value of velocity is conserved for a billiard, we may normalize the velocity as a unit vector and then let $v \in[0,1]$. The linearized Poincaré map from $\left(s_{1}, v_{1}\right)$ to $\left(s_{2}, v_{2}\right)$ can be expressed as [11, 12, 13]

$$
\begin{aligned}
M(12) & =\left(\begin{array}{cc}
\left(l_{12} c_{1}-v_{1 \perp}\right) / v_{2 \perp} & -l_{12} / v_{1 \perp} v_{2 \perp} \\
c_{1} v_{2 \perp}+c_{2} v_{1 \perp}-l_{12} c_{1} c_{2} & \left(l_{12} c_{2}-v_{2 \perp}\right) / v_{1 \perp}
\end{array}\right) \\
& =\left(\begin{array}{cc}
1 / v_{2 \perp} & 0 \\
0 & v_{2 \perp}
\end{array}\right)\left(\begin{array}{cc}
1 & 0 \\
-c_{2} / v_{2 \perp} & 1
\end{array}\right)\left(\begin{array}{cc}
-1 & -l_{12} \\
0 & -1
\end{array}\right)\left(\begin{array}{cc}
1 & 0 \\
-c_{1} / v_{1 \perp} & 1
\end{array}\right)\left(\begin{array}{cc}
v_{1 \perp} & 0 \\
0 & 1 / v_{1 \perp}
\end{array}\right)
\end{aligned}
$$

where $l_{12}$ is the length of the chord joining $s_{1}$ and $s_{2}, v_{\perp}$ is the normal component of the velocity and $c$ the curvature of the billiard boundary. It is often useful to know the Jacobian matrix $\partial(s, v) / \partial(\xi, \kappa)$, where $\xi$ and $\kappa$ are perturbations in displacement and velocity at a given point $\mathrm{O}$ on the straight line joining $s_{1}$ and $s_{2}$ along the direction perpendicular to the path. Without loss of generality we may choose the coordinate 
system with the origin at $\mathrm{O}$, and the $y$-axis along the path from $s_{1}$ to $s_{2}$, as shown in Fig. 2. (In the figure $s_{1}$ and $s_{2}$ are marked as $s$ and $s^{\prime}$, respectively.) It is obvious that at $s_{1}$

$$
v_{x}=0, \quad v_{y}=1 .
$$

Denote by a dot the derivative with respect to the arc length $s$. The tangent and normal unit vectors are $\mathbf{t}_{1}=\left(\dot{x}_{1}, \dot{y}_{1}\right)$ and $\mathbf{n}=\left(-\dot{y}_{1}, \dot{x}_{1}\right)$, respectively. This implies that

$$
v_{1}=\dot{x}_{1} \quad \text { and } \quad v_{1 \perp}=\dot{y}_{1} .
$$

Assume that a perturbation $(\delta s, \delta v)$ from $s_{1}$ to $\tilde{s}=s_{1}+\delta s$ results in the perturbation at $\mathrm{O},(\xi, \kappa)$. Up to the lowest order, we have

$$
\tilde{x} \equiv x(\tilde{s})=x_{1}+\dot{x}_{1} \delta s=v_{1 \perp} \delta s \quad \text { and } \quad \tilde{y} \equiv y(\tilde{s})=y_{1}+\dot{y}_{1} \delta s \approx y_{1} .
$$

It can be seen that $\delta v_{1 x} \equiv \tilde{v}_{x}-v_{1 x}=\kappa$. From Eq. ( 30 ), the relation $v_{x}\left(\delta v_{x}\right)+v_{y}\left(\delta v_{y}\right)=0$ implies that $\delta v_{1 y}=0$. That is, at $\tilde{s}$ we have $\tilde{v}_{x}=\kappa$, and $\tilde{v}_{y}=v_{1 y}=1$. Using $\tilde{v}_{x} / \tilde{v}_{y}=(\tilde{x}-\xi) / \tilde{y}$, we find

$$
v_{1 \perp} \delta s_{1}-\xi=y_{1} \kappa .
$$

Similarly, the relation $v=v_{x} \dot{x}+v_{y} \dot{y}$ leads to

$$
\delta v_{1}=v_{1 \perp} \kappa+\ddot{y} \delta s=v_{1 \perp} \kappa+c_{1} v_{1 \perp} \delta s,
$$

where we have used the curvature formula $\ddot{y}=c \dot{x}$. Equations (32) and (33) can be written as

$$
\left(\begin{array}{l}
\delta s_{1} \\
\delta v_{1}
\end{array}\right)=\left(\begin{array}{cc}
1 / v_{1} \perp & y_{1} / v_{1 \perp} \\
c_{1} & v_{1 \perp}+c_{1} y_{1}
\end{array}\right)\left(\begin{array}{l}
\xi \\
\kappa
\end{array}\right) .
$$

The above transform matrix may be written as

$$
J_{s \xi}=\left(\begin{array}{cc}
1 / v_{1} \perp & 0 \\
0 & v_{1 \perp}
\end{array}\right)\left(\begin{array}{cc}
1 & 0 \\
c_{1} / v_{1} \perp & 1
\end{array}\right)\left(\begin{array}{cc}
1 & y_{1} \\
0 & 1
\end{array}\right) .
$$

Its inverse

$$
J_{\xi s} \equiv J_{s \xi}^{-1}=\left(\begin{array}{cc}
1 & -y_{1} \\
0 & 1
\end{array}\right)\left(\begin{array}{cc}
1 & 0 \\
-c_{1} / v_{1 \perp} & 1
\end{array}\right)\left(\begin{array}{cc}
v_{1 \perp} & 0 \\
0 & 1 / v_{1 \perp}
\end{array}\right),
$$

describes the tranformation from $\left(\delta s_{1}, \delta v_{1}\right)$ to $(\xi, \kappa)$.

Along similar lines we may derive the Jacobian matrices between $\left(\delta s_{2}, \delta v_{2}\right)$ and $(\xi, \kappa)$. There is a main difference. When following the above derivation for $s_{2}$, we use $v_{x}$ and $v_{y}$ of the velocity right before a bounce. By taking this into account, $v_{\perp}$ in the above formulas has to be replaced by $-v_{\perp}$. For example,

$$
\left(\begin{array}{l}
\delta s_{2} \\
\delta v_{2}
\end{array}\right)=\left(\begin{array}{cc}
-1 / v_{2 \perp} & -y_{2} / v_{2 \perp} \\
c_{2} & -v_{2 \perp}+c_{2} y_{2}
\end{array}\right)\left(\begin{array}{c}
\xi \\
\kappa
\end{array}\right),
$$

and

$$
J_{s \xi}\left(s_{2}\right)=\left(\begin{array}{cc}
-1 / v_{2 \perp} & 0 \\
0 & -v_{2 \perp}
\end{array}\right)\left(\begin{array}{cc}
1 & 0 \\
-c_{2} / v_{2} \perp & 1
\end{array}\right)\left(\begin{array}{cc}
1 & y_{2} \\
0 & 1
\end{array}\right) .
$$

Noting that $l_{12}=y_{2}-y_{1}$, we can verify that

$$
M(12)=J_{s \xi}\left(s_{2}\right) J_{\xi s}\left(s_{1}\right) .
$$

For an orbit which starts and ends inside the billiard, respectively, at $\left(\mathbf{r}_{0}, \mathbf{p}_{0}\right)$ and $\left(\mathbf{r}_{t}, \mathbf{p}_{t}\right)$, and makes successive bounces at $s_{1}, s_{2}, \ldots, s_{n}$ in between, we have

$$
\left(\begin{array}{c}
\xi_{t} \\
\kappa_{t}
\end{array}\right)=\mathcal{M}\left(\begin{array}{c}
\xi_{0} \\
\kappa_{0}
\end{array}\right), \quad \mathcal{M}=J_{\xi s}\left(s_{n}\right) M(n-1, n) \cdots M(12) J_{s \xi}\left(s_{1}\right) .
$$


The Jacobian $\partial \xi_{t} / \partial \kappa_{0}$ is determined as

$$
\partial \xi_{t} / \partial \kappa_{0} \equiv k \partial r_{\perp} / \partial p_{\perp}^{\prime}=\mathcal{M}_{12}
$$

where $k$ is the absolute value of the conserved momentum. When all the bounces happen at straight segments of the boundary with curvature $c=0$, matrix $\mathcal{M}$ is significantly simplified. In this case, using expressions (39) and (36), we have

$$
\mathcal{M}=\left(\begin{array}{cc}
1 & \left|y_{n}\right| \\
0 & 1
\end{array}\right)\left(\begin{array}{cc}
-1 & l_{n-1, n} \\
0 & -1
\end{array}\right) \cdots\left(\begin{array}{cc}
-1 & l_{12} \\
0 & -1
\end{array}\right)\left(\begin{array}{cc}
1 & \left|y_{1}\right| \\
0 & 1
\end{array}\right)=(-1)^{n}\left(\begin{array}{cc}
1 & L \\
0 & 1
\end{array}\right),
$$

where $L$ is the total length of the orbit. Thus, from Eq. (40) we have

$$
\partial r_{\perp} / \partial p_{\perp}^{\prime}=(-1)^{n} L / k
$$

for the Jacobian.

The author thanks Drs. Y. Gu, Baowen Li and A. M. Ozorio de Almeida for useful discussions. This work was supported in part by the National Natural Science Foundation of China.

\section{References}

[1] H. Weyl, Nachr. Akad. Wiss. Göttingen (1911) 110.

[2] R. Balian and C. Bloch, Ann. Phys. (NY) 60 (1970) 401; 64 (1971) 271.

[3] H. P. Baltes and E. R. Hilf, Spectra of Finite Systems (B.I.-Wissenschaftsverlag, Mannheim, 1976) and references therein.

[4] M. Kac, Am. Math. Monthly, 73 (1966) 1.

[5] M. V. Berry and K. E. Mount, Rep. Prog. Phys. 35 (1972) 315.

[6] M. C. Gutzwiller, Chaos in Classical and Quantum Mechanics (Springer, New York, 1990); L. E. Reichl, The Transition to Chaos in Conservative Classical Systems: Quantum Manifestations (Springer, New York 1992).

[7] M. Brack and R. K. Bhaduri, Semiclassical Physics (Addison-Wesley, Reading, 1997).

[8] M. V. Berry and M. Tabor, Proc. R. Soc. Lond. A349 (1976) 101.

[9] V. M. Strutinsky and A. G. Magner, Sov. J. Part. Nucl. 7 (1976) 138.

[10] A. M. Ozorio de Almeida and J. H. Hannay, J. Phys. A20 (1987) 5873.

[11] M. V. Berry, Eur. J. Phys. 2 (1981) 91.

[12] M. Sieber and F. Steiner, Physica D44 (1990) 248.

[13] H. R. Dullin, P. H. Richter and A. Wittek, Chaos 6 (1996) 43.

Fig. 1 Images of a given point $Q$ in a corner. Some mediate point $Q^{\prime}$ is used to construct folded paths. Fig. 2 Perturbation of a path. 


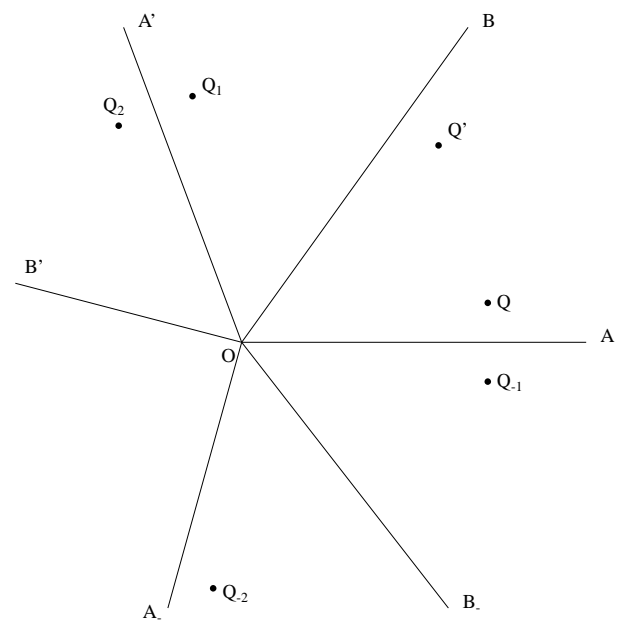




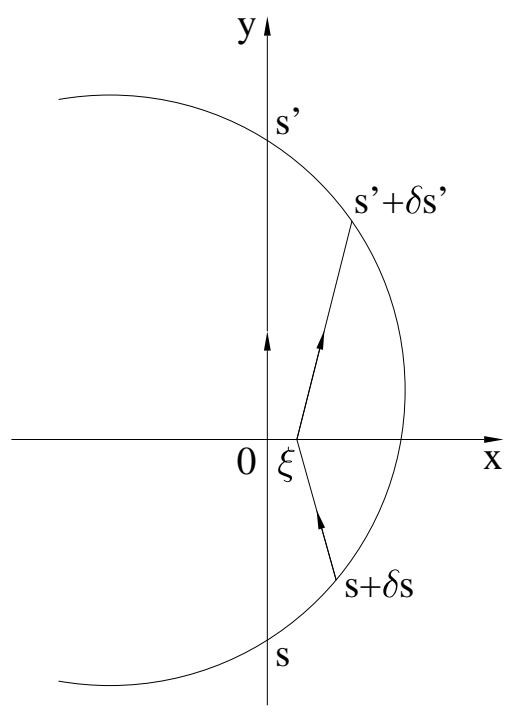

